\title{
Polyvinylidene difluoride-based composite: glassy dynamics and pretransitional behaviour
}

\author{
Szymon Starzonek ${ }^{1, a}$, Kena Zhang ${ }^{2}$, Aleksandra Drozd-Rzoska ${ }^{1}$, Sylwester J. Rzoska ${ }^{1}$, \\ Emilia Pawlikowska ${ }^{1}$, Mikolaj Szafran ${ }^{3}$, and Feng Gao ${ }^{2}$ \\ ${ }^{1}$ Institute of High Pressure Physics of the Polish Academy of Sciences, ul. Sokolowska 29/37, 01-142 Warsaw, \\ Poland \\ 2 State Key Laboratory of Solidification Processing, MIIT Key Laboratory of Radiation Detection Materials and \\ Devices, NPU-QMUL Joint Research Institute of Advanced Materials and Structure, School of Material Science \\ and Engineering, Northwestern Polytechnical University, Xi'an 710072, P.R. China \\ 3 Department of Chemistry, Warsaw University of Technology, Warsaw, Poland
}

Received 4 March 2019 / Received in final form 8 November 2019

Published online 23 March 2020

(C) The Author(s) 2020. This article is published with open access at Springerlink.com

\begin{abstract}
This paper presents results of broadband dielectric spectroscopy studies in the composite system for which particularly strong interactions between polyvinylidene difluoride (PVDF: ferroelectric polymer, $\left.T_{C}=453-473 \mathrm{~K}\right)$ matrix and barium strontium titanate (BST) ferroelectric micro-particles can be expected. For PVDF the super-Arrhenius (SA) dynamics, associated with segmental motions freezing at the glass temperature $T_{g}=235 \mathrm{~K}$, is evidenced. The addition of BST particles qualitatively changes dynamics, converting the SA-type behaviour in PVDF to the clear Arrhenius one in BST/PVDF composite. The latter crossovers to the relaxor-type SA dynamics on cooling, exactly at the glass temperature of PVDF. The preliminary model explaining such unique behaviour is proposed. For the consistent portraying of the SA evolution of primary relaxation times in PVDF and BST/PVDF, the activation energy index analysis was carried out and the new equation, entropy and symmetry controlled, is introduced. Studies are accomplished by the analysis of the ferroelectric-paraelectric transition in PVDF and for the composite system. They led to the discovery of the strong pretransitional anomaly of $d \varepsilon / d T$, extending even to the vicinity of the room temperature, The semi-discontinuous nature of melting in PVDF and its composites, with the discontinuity metric $\triangle T \approx 20 \mathrm{~K}$ is suggested.
\end{abstract}

\section{Introduction}

The glass transition is one of the grand challenges of the solid-state physics, promising the fundamental breakthrough $[1,2]$ matched with possible qualitative progress in many branches of materials engineering [2-4]. The key attractor for researchers constitutes 'universal' previtreous phenomena for dynamic properties, shared amongst microscopically distinct systems and starting even $100 \mathrm{~K}$ above the glass temperature $T_{g}$. One can recall the super-Arrhenius (SA) evolution of the primary relaxation time, the broadening of its distribution on cooling or the translational-orientational decoupling [2]. Still less examined is the solid, amorphous state below $T_{g}[2-4]$. Each year the application of innovative experimental and analytic methods increases the knowledge on the glass transition, but the long-expected cognitive breakthrough has been not reached yet [1-4]. Consequently, focused studies beyond dominant paths can have a milestone meaning for approaching the ultimate insight into this

\footnotetext{
${ }^{a}$ e-mail: szymon.starzonek@wp.pl
}

puzzling topic. A possible milestone direction can offer studies in 'innovative' glass forming systems, such as composite materials benefiting from the combination of a polymeric glass matrix and solid nano- or microparticle [5-16]. This can lead to qualitatively new glassy metamaterial features, results for the added value combination of constituents' properties [14-16].

This report presents results of broadband dielectric spectroscopy studies in the composite system for which a particularly strong interaction between the polymer matrix host (polyvinylidene fluoride, PVDF) and barium strontium titanate (BST) ferroelectric micro-particles can be expected. For PVDF the super-Arrhenius (SA) behaviour of the primary relaxation time, associated with segmental motions freezing at the glass temperature $T_{g}=$ $235 \mathrm{~K}[6,7]$, is evidenced. It is shown that the addition of BST particles qualitatively changes dynamics, converting the SA-type dynamics in PVDF to Arrhenius one in BST/PVDF composites. The latter crossovers to the relaxor-type SA dynamics on cooling exactly at the glass temperature of PVDF. The preliminary explanation of this behaviour is proposed. 
Studies focused on dynamics are accomplished by the analysis of the ferroelectric-paraelectric transition in PVDF $\left(T_{c}=453-473 \mathrm{~K}\right)$ and a polymer-solid particles composite. They led to the finding of the strong pretransitional anomaly of $d \varepsilon / d T$, extending even to the vicinity of the room temperature, and the suggestion of the semi-discontinuous nature of melting in PVDF and its composites. We stress the importance of the insight into fundamental properties of PVDF and its composites, due to the enormous and developing range of applications.

\section{Experimental}

\subsection{Preparation of samples}

Studies were carried out for PVDF $+x$ BST (labeled as BST/PVDF) composites, where $x$ is for $10 \%, 20 \%, 30 \%$, and $40 \%$ volume fractions, respectively. $\mathrm{Ba}_{0.6} \mathrm{Sr}_{0.4} \mathrm{TiO}_{3}$ (BST) powders were fabricated by the solid-state reaction method employing the raw materials of $\mathrm{BaCO}_{3}, \mathrm{SrCO}_{3}$ and $\mathrm{TiO}_{2}$ which were mixed for the stoichiometric composition and heated at $1673 \mathrm{~K}$ for $2 \mathrm{~h}$, with repeated grinding. For BST particles, the pure perovskite structures were detected. The analysis of their size indicated the dominated impact of $D_{50}=0.478 \mu \mathrm{m}, D_{90}=1.474 \mu \mathrm{m}$ grains. The average particle size was $0.6 \mu \mathrm{m}$, as estimated using the Laser Diffraction Particle Size Analyzer.

For fabricating BST/PVDF composites, the Poly(VinyliDene Fluoride, PVDF) (FR904) was supplied by Shanghai 3F New Materials Co., Ltd. N,N-dimethylformamide (DMF, anhydrous 99.8\%) was purchased from Sinopharm Chemical Reagent Co., Ltd. Silane coupling agent KH550 modified the surface of BST particles to enhanced the connectivity between BST fillers and PVDF matrix. The PVDF and BST powders were mixed in a DMF solvent. The mixture was ultrasonically dispersed for $2 \mathrm{~h}$ and stirring for $6 \mathrm{~h}$. Subsequently, it was tape-casted on a glass substrate to obtain a thick film. After that, the film was kept in a vacuum oven overnight at $333 \mathrm{~K}$ to evaporate the organic solvent of DMF. Then the BST/PVDF films with $0.1-0.2 \mathrm{~mm}$ thickness was produced by hot pressing $(P=100 \mathrm{MPa})$ at $T=393 \mathrm{~K}$ for $2 \mathrm{~h}$. BST powders were dispersed uniformly within the polymer matrix without visible defects. The high compatibility of BST fillers and PVDF matrix derives from the forming of hydrogen bonds between the $-\mathrm{NH}_{2}$ groups on the surface of the particles and the $\mathrm{F}$ atoms of the PVDF molecular chains. More details about the fabrication and the microstructure can be found in reference [10].

\subsection{Broadband dielectric spectroscopy measurements}

The broadband dielectric spectroscopy (BDS) is the basic research method for studying dynamics in glass forming systems due to its unique possibility of the fast impedance scan covering up to 15 decades in frequency/time $[2,17]$. Results can be presented and analyzed in dielectric permittivity, modulus, and conductivity representations, to spotlight on specific features of the tested system
$[2,17,18]$. This report focuses on dielectric permittivity, namely: (i) its imaginary part contains messages related to orientational and translational relaxation times [17] (ii) the real part enables the insight into the dipoledipole arrangements [17], what is essential for studies on phase transitions and pretransitional effects. Experimental studies were carried out using the Novocontrol BDS spectrometer, which can operate for frequencies between $3 \mu \mathrm{Hz}$ and $3 \mathrm{GHz}$, with very high resolution. Samples were placed in flat-parallel capacitors with gaps between plates $d=0.2 \mathrm{~mm}$ and $d=1 \mathrm{~mm}$, diameters $10 \mathrm{~mm}$ and $20 \mathrm{~mm}$. The measuring voltage $U=1 \mathrm{~V}$ was applied. In the solid state of samples, the capacitor had the form of the silver film applied directly to the surface of the material, to avoid any impact of pressure introduced by the design of the capacitor. The temperature control was assured thanks to the Novocontrol Quattro System, with $\pm 0.02 \mathrm{~K}$ accuracy.

\section{Methods}

\subsection{The activation energy temperature index in glass forming systems}

One of key hallmarks of the previtreous behaviour for $T \rightarrow$ $T_{g}$ in glass-forming systems is the Super-Arrhenius (SA) increase of the primary (structural, alpha) relaxation time $[2,17,18]$ :

$$
\tau(T)=\tau_{0} \exp \left(\frac{E_{a}(T)}{R T}\right)
$$

where $T>T_{g}$ and $E_{a}(T)$ is the apparent (temperature dependent) activation energy. For $E_{a}(T)=E_{a}=$ const one obtains the basic Arrhenius (A) dependence. $\tau_{0}$ is the pre-factor.

The glass temperature is 'stretched' in some temperature range and its value depends on the cooling rate $[2,17,18]$. Consequently, it cannot be directly included into the phase transitions pattern. The most classical ways of estimating $T_{g}$ is based on the heat capacity scan, with the $10 \mathrm{~K} / \mathrm{min}$ cooling rate $[2,17,18]$. The obtained in this 'thermodynamic' estimation of $T_{g}$ is in fair agreement with the 'dynamic' based on the empirical assumption $\tau\left(T_{g}\right)=100 \mathrm{~s}$. The latter is associated with the far previtreous effect (slowing down) of $\tau(T)$, starting even $100 \mathrm{~K}$ above $T_{g}[2,17]$. This 'anomalous' slowing down is different for various glass formers and classified using the fragility coefficient $m=\left[d \log \tau(T) / d \log \left(T_{g} / T\right)\right]_{T=T_{g}}$. Its values range between $m \sim 16$ for the almost-Arrhenius behaviour and $m \sim 200$ for the extremely Super- Arrhenius (SA) ones $[2,17-20]$. For describing the previtreous behaviour of dynamic properties the SA equation (6) cannot be directly applied due to the unknown general form of the apparent activation energy and ersatz relations are used. The basic one is the Vogel-Fulcher Tamman (VFT) equation (15) $[2,17,18]$. There are other basic modelequations (MYEGA, AM, etc.) which can be used for portraying $\tau(T)$ experimental data, yielding a similar fitting reliability. However, forms of $I_{D O}(T)$ indexes associated with these relations are qualitatively different, what 
Table 1. Forms of the index of energy.

\begin{tabular}{llll}
\hline $\begin{array}{l}\text { Equation } \\
\text { name }\end{array}$ & Equation form & $1 / I_{D O}(T)$ & Parameter $n$ \\
\hline VFT & $\tau(T)=\tau_{0} \exp \left(\frac{D_{T} T_{0}}{T-T_{0}}\right)$ & $I_{D O}^{-1}=\left(\frac{1}{T_{0}}\right) T-1$ & $n=1$ \\
{$[2,24-26]$} & $\tau(T)=\tau_{0}$ & $I_{D O}^{-1}=\left(\frac{1}{C}\right) T$ & $n=0$ \\
WM, & $\exp \left(\frac{K}{T} \exp \left(\frac{C}{T}\right)\right)$ & \\
MYEGA & & \\
{$[27,28]$} & $\tau(T)=\tau_{0} \exp \left(\frac{B}{T^{D}}\right)$ & $I_{D O}^{-1}=\frac{1}{D-1}=$ const & $\left.n=\frac{1}{1-D}{ }^{*}\right)$ \\
Avramov, & & \\
AM & & \\
{$[29-31]$} & & \\
\hline * Reported results indicate that $D>1$ for the AM equation $[2]$ and then $n<0$.
\end{tabular}

offers the decisive route for the validation [21-23]. This is summarised in Table 1.

The most common way of comparing the reliability of the given descriptions is the residual analysis or the linearised derivative-based plots which showed the prevalence of the AM or MYEGA relations for some low molecular weight glass formers. For plastic crystals and liquid crystals, the prevalence of the critical-like portrayal was proved. Notwithstanding, the advantages over the VFT description were subtle and it could serve as the alternative, effective way of portraying. The decisive way of comparing the fundamental validity of different equations offers the analysis of the apparent activation energy temperature index [21-23]:

$$
I_{D O}=-\frac{d \ln E_{a}(T)}{d \ln T}=\frac{d \ln E_{a}(T) / E_{a}(T)}{d \ln T / T} .
$$

As shown in Table 1 , forms of $1 / I_{D O}(T)$ strongly differ for each mentioned equation.

To calculate experimental values of $I_{D O}(T)$ the protocol for the non-biased determining of the apparent activation energy without the knowledge of $\tau_{0}$ pre-factor in prior, was developed. It bases on the numerical solution, for the given set of $\tau(T)$ data, of the differential equation resulted from the general SA equation (15) [21-23]:

$$
\frac{d E_{a}^{\prime}(T)}{d(1 / T)}+\frac{E_{a}^{\prime}(T)}{(1 / T)}=\frac{H_{a}^{\prime}(T)}{(1 / T)}
$$

where $E_{a}^{\prime}(T)=E_{a}(T) / R, H_{a}^{\prime}(T)=H_{a}(T) / R$ and the apparent activation enthalpy is calculated from $H_{a}^{\prime}(T)=$ $d \ln \tau(T) / d(1 / T)$.

As shown in references [21-23] for almost 50 different glass formers the reciprocal of the index exhibits the 'universal' linear behaviour (Eq. (16)). The index $I_{D O}(T)$ is directly related to basic process energies and fragility [23]:

$$
I_{D O}(T)=\frac{\triangle H_{a}(T)}{\triangle E_{a}(T)}-1
$$

$m=C\left(1+I_{D O}\left(T_{g}\right)\right)=\left(2-\log \tau_{0}\right)\left[1+I_{D O}\left(T_{g}\right)\right]$ and

$m \approx 16\left[1+I_{D O}\left(T_{g}\right)\right]$.
The latter approximated relation is for $\tau_{0}=10^{-14} \mathrm{~s}$, often assumed as the general 'reference' value for prefactors $[2,32]$. It is notable that in practice for different glass formers $t_{0}$ ranges between $\tau_{0}=10^{-10}$ and $\tau_{0}=$ $10^{-16}[21,27,31,33]$.

\section{Results}

\subsection{New features for the dielectric characterization of BST ceramic (guest) and PVDF polymer (host)}

The most common and general analytic routine for complex dielectric permittivity spectra employs the HavriliakNegami function $[2,17]$ :

$$
\varepsilon^{*}(\omega)=\frac{\sigma}{\omega \varepsilon_{0}}+\varepsilon_{\infty}+\frac{\triangle \varepsilon}{\left(1+(i \omega \tau)^{\alpha}\right)^{\beta}}
$$

where $\triangle \varepsilon=\varepsilon_{s}-\varepsilon_{\infty}$ is the dielectric strength, $\omega=2 \pi f$, $\tau$ describes the distribution of relaxation times: for $\alpha=1$ and $\beta=1$ one obtains the basic Debye relation; $\sigma$ stands for DC electric conductivity.

The application of equation (6) yields the average value of the (primary) relaxation times for the given temperature (or pressure) and parameters describing their distribution $[2,17]$. However, equation (6) is associated with 6 adjustable parameters and the non-linear fitting routine, which introduces a notable uncertainty. Such analysis strongly depends on the onset values of parameters and the assumed ad hoc range of validity of the given description. To avoid these 'potentially parasitic' factors, the different analytic routine for experimental data was used. In 1983 Jonscher reported the "universal relaxation law' for $\varepsilon^{\prime \prime}(f)$ loss curves in solids, namely [34]:

$$
\log \varepsilon^{\prime \prime}(f)=m \log f \quad \text { for } f<f_{\text {peak }}
$$

$$
\log \varepsilon^{\prime \prime}(f)=-n \log f \quad \text { for } f>f_{\text {peak }} .
$$

For the single relaxation time Debye-type process two branches of the loss curve are described by $m=1$ and $n=1$. It is worth recalling, that coefficients in equation (6) and equations (7) and (8) are interrelated: $m=\alpha$ and $m=\alpha \beta[2,17,34]$. Basing on the empirical behaviour 
described by equations (7) and (8) one can estimate the relaxation time from the peak frequency of the loss curve via the condition:

$$
\frac{d \log \varepsilon^{\prime \prime}(f)}{d \log f}=0 \quad \text { for } f=f_{\text {peak }} \text { and } T=\text { const }
$$

where $\tau=1 / 2 \pi f_{\text {peak }}$.

The application of equations (7)-(9) estimates relaxation times $\tau(T)$ and their distribution shape parameters based solely on the transformation of experimental data, avoiding the multiparameter fitting. Generally, there are two ways of presentation of BDS experimental data. In the Glass Transition Physics $\varepsilon^{\prime}(f)$ and $\varepsilon^{\prime \prime}(f)$ spectra for different temperatures are plotted and analyzed $[2,18]$. In the Physics of Ferroelectrics, for instance, commonly applied are plots of the real part of dielectric permittivity $\varepsilon^{\prime}(T)$ and dielectric loss $\tan \delta(T)=\varepsilon^{\prime \prime} / \varepsilon^{\prime}$ for a constant measurement frequency [35]. There are no reports regarding the 'universal form' of $\tan \delta(T)$ dependences, to the best of the authors' knowledge. However, such behaviour is evidenced below, both for BST and BST/PVDF composites, namely:

$$
\begin{aligned}
& \tan \delta(T)=C \exp \left( \pm b_{ \pm} T\right) \text { and then } \\
& \ln \tan \delta(T)=\ln C \pm b_{ \pm} T \text { for } f=\text { const }
\end{aligned}
$$

where ' $(+)$ ' is referred to $T<T_{\text {peak }}$ and '(-)' for $T>$ $T_{\text {peak }}$.

Consequently, one can determine $T_{\text {peak }}$ from the condition:

$$
\frac{d \ln \tan \delta(T)}{d T}=0 \text { for } T=T_{\text {peak }} \text { and } f=\text { const } .
$$

The relaxation time for the given temperature is determined as $\tau(T)=\tau\left(T_{\text {peak }}\right)=1 / 2 \pi f$, where $f$ is the measurement frequency used for the temperature scan.

Figures 1a-1c present the 'discussion' of the temperature behaviour of $\varepsilon^{\prime}(T)$ for BST ceramic applied for the preparation of BST/PVDF composites. Figure 1a presents experimental behavior of the real part of dielectric permittivity for frequencies related to the static domain of dielectric constant $\left(\varepsilon^{\prime} \approx \varepsilon\right)$. The simple analysis of these data, shown in the insets in Figure $1 \mathrm{~b}$ reveals the Curie-Weiss (CW) type behaviour $\varepsilon \propto 1 /\left|T-T_{C}\right|$ [35], namely: $1 / \varepsilon=a \pm b T$ and then $\varepsilon(T)=1 /(a \pm b T)=$ $(1 / b) /(a / b \pm T)$. It is notable that the CW-type domain is detected also well beyond $T_{C} \approx 286 \mathrm{~K}$, associated with the paraelectric-ferroelectric transition. In the crossover region between 'close-to- $T_{c}$ ' and 'remote-from- $T_{c}$ ' $\mathrm{CW}$ domains: $\frac{1}{\varepsilon}=a^{\prime}+b^{\prime} T \pm c^{\prime} T^{2}$.

The main part of Figure 1c presents the typical plot of the imaginary part of dielectric loss applied for such materials as BST. The same data are shown in the inset using the semi-log plot, revealing the functional pattern of the behaviour:

$$
\ln \tan \delta(T)=a \pm b_{ \pm} T
$$

where ' $(+)$ ' is referred to $T<T_{\text {peak }}$ and ' $(-)$ ' for $T>$ $T_{\text {peak }}$.

Consequently, one obtains:

$$
\begin{aligned}
\tan \delta(T) & =\exp \left(a \pm b_{ \pm} T\right)=\exp (a) \exp \left( \pm b_{ \pm} T\right) \\
& =C \exp \left( \pm b_{ \pm} T\right)
\end{aligned}
$$

where $C=\exp (a)=$ const.

PVDF applied for the research described in the given report melted at $T_{m} \approx 433 \mathrm{~K}$ and remained in the stable fluid phase up to ca. $480 \mathrm{~K}[5,10]$. The solid phase below $T_{m}$ is associated with notable freedom of segmental motions, leading to the complex 'glassy' dynamics resembling ones observed in supercooled, ultraviscous liquids [2]. The final solidification occurs at $T_{g} \approx 235 \mathrm{~K}$ [5].

Figure $2 \mathrm{a}$ presents the temperature dependence of $\varepsilon^{\prime}(f)$, from the near-room temperature up to $\sim T_{m}$. The low-temperature domain for PVDF and BST/PVDF composites is discussed in the subsequent section for selected frequencies. The frequency $f=1 \mathrm{kHz}$ can be considered as the static one and $\left(\varepsilon^{\prime}=\varepsilon\right)$, i.e., it is related to dielectric constant. Notable is the strong increase of $\varepsilon(T)$ on heating towards $T_{m}$, which may seem to follow the (almost) linear behaviour in Figure 2a.

Such a behavior usually explained as the consequence of strengthening of dipole related molecular motion associated with $-\mathrm{C}-\mathrm{F}$ molecular group, with a hypothetically linear dependence $\varepsilon(T) \approx a+b T$ [10]. However, the derivative plot $d \varepsilon / d T$ shown in the inset, reveals the complex behaviour of $(T)$, which can be portrayed by the relation:

$$
\frac{d \varepsilon}{d T}(T)=C+A\left(T-T^{*}\right)^{-\phi}
$$

where $T^{*}=T_{m}+\triangle T^{*}, \triangle T^{*} \approx 25 \mathrm{~K}$ and the exponent $\phi=4.2 \pm 1$.

Generally, melting is considered as the clear example of the first order (discontinuous) phase transition with a 'sudden and sharp' discontinuous change of physical properties, such as density heat capacity, dielectric constant and no pretransitional effects [36]. So far, the puzzle of the phase transitions in solids has been arranged/solved only for the continuous phase transitions, where universal pretransitional ('pre-critical') anomalies are the key feature $[37,38]$. The emergence of the Physics of Critical Phenomena is considered as one of the grand successes of the condensed matter physics in the $20^{\text {th }}$ Century [36,38]. However, there are also intermediate cases of 'weakly' discontinuous phase transitions where the pretransitional, critical-like, behavior terminates at the discontinuous 'melting/freezing' temperature $T_{m}$ before reaching the (hypothetical) 'critical' temperature $\left(T^{*}, T_{C}\right)$ [38]. Such a behavior directly leads to the metric of the discontinuity of the 'weakly discontinuous' phase transitions $\triangle T^{*}=T^{*}-T_{m}$. The most known examples are the Isotropic-Nematic or Isotropic-Smectic phase transitions in liquid crystals, with $\triangle T^{*}$ ranging from $0.7 \mathrm{~K}$ to $15 \mathrm{~K}$ where freezing of selected single element of (orientational) symmetry takes place [38-41]. Equation (14) seems to follow the latter pattern, although the question 

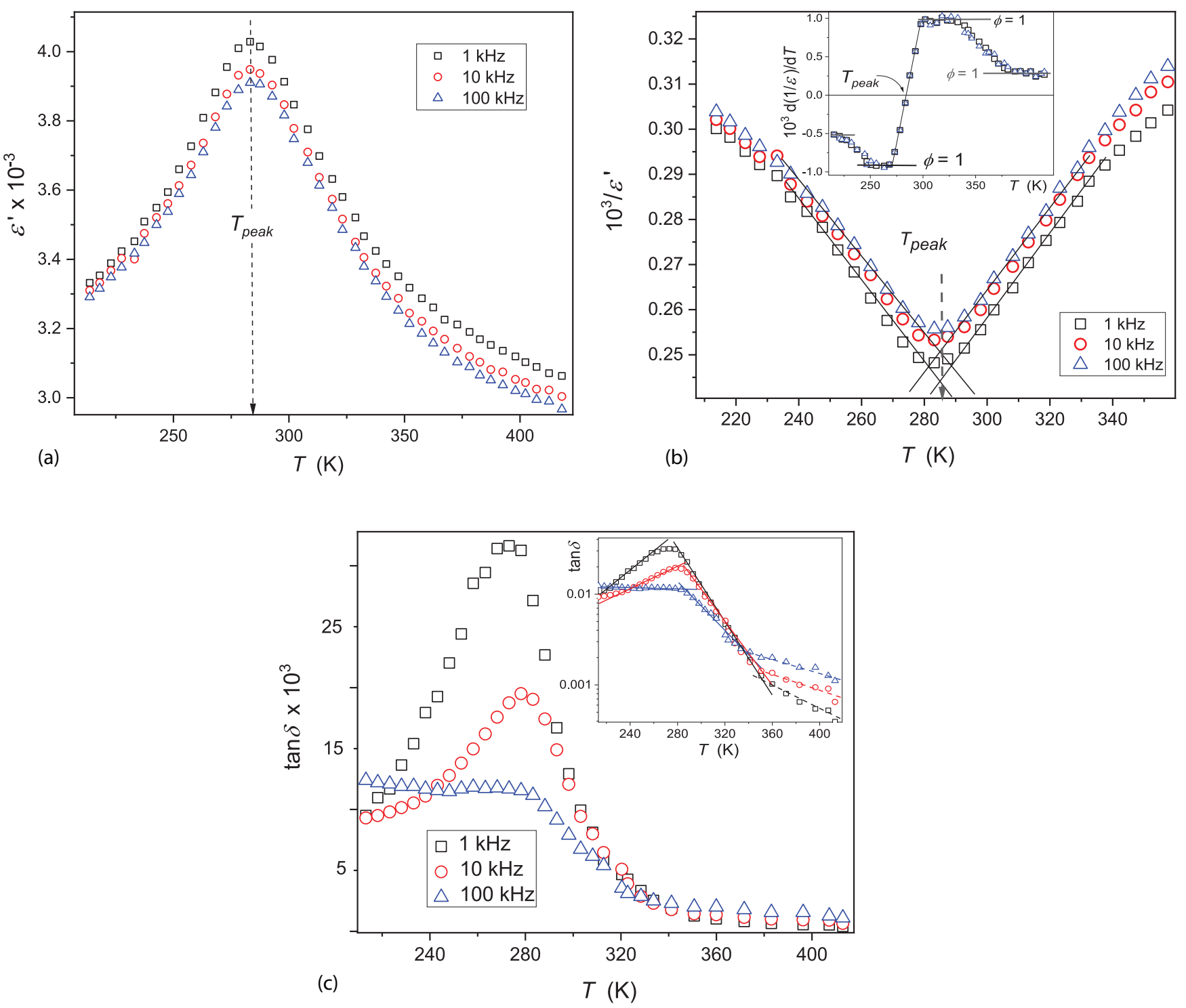

Fig. 1. (a) The temperature evolution of the real part of dielectric permittivity for the applied BST ceramic. (b) The reciprocal of the real part of dielectric permittivity in the surrounding of the peak visible in Figure 1 (BST ceramic). Straight lines reveal the Curie-Weiss (CW) type behaviour; the arrow indicates the para/ferro critical temperature $T_{C} \approx 286 \mathrm{~K}$. The inset shows the derivative of data from the main part of the plot, but in full tested temperature range. Horizontal lines in the inset, with the 'exponent $\phi=1$, show the real extension of the Curie-Weiss type behaviour. (c) The temperature behaviour of dielectric loss in the tested BST ceramic. The inset shows the same data in the semi-log scale revealing the functional form of the evolution.

of the obtained 'critical' exponent $=4$ remains. In the opinion of the authors, this can be associated with the ferro- or paraelectric nature of PVDF [5-9]. Studies of dielectric permittivity (dielectric constant) in the paraelectric phase of crystals such as triglycine sulphate for increasing electric field showed that for $\left(T \rightarrow T_{C}\right)$ [42]: $\varepsilon \propto$ $1 /\left(T \rightarrow T_{C}\right)$ and $\triangle \varepsilon^{E} / E^{2} \propto 1 /\left(T \rightarrow T_{C}\right)^{4}$. Dielectric constant $(\varepsilon)$ detects the averaged arrangement of permanent dipole moments [36], but $\triangle \varepsilon^{E}$ is directly coupled to local fluctuations of the order parameter $\left\langle\triangle M^{2}\right\rangle_{V}$ and then multimolecular ordered 'heterogeneities-fluctuations' in the 'fluidlike' (chaotic) surrounding [43]. Recently, it was indicated in reference [44] that for 'glassy' systems the peculiar temperature dependence of the variance of the dipole moment of a polar liquid has its origin in the three-point static correlation function, which accommodates three- and four-dipole microscopic correlations in the supercooled previtreous state [44], and then $d \varepsilon / d T \propto$ $\left\langle\triangle M^{2}\right\rangle_{V} \propto \Delta \varepsilon^{E}$. All these can point out sources of the unusual behavior $d \varepsilon / d T \sim 1 /\left(T-T^{*}\right)^{4}$ for $T \rightarrow T_{m}$, in the paraelectric domain of PVDF polymer.

\subsection{New features BST/PVDF composites emerging from BDS studies}

BDS scans were carried out for PVDF and BST/PVDF composites with $10-40 \%$ volume fractions of BST. For 

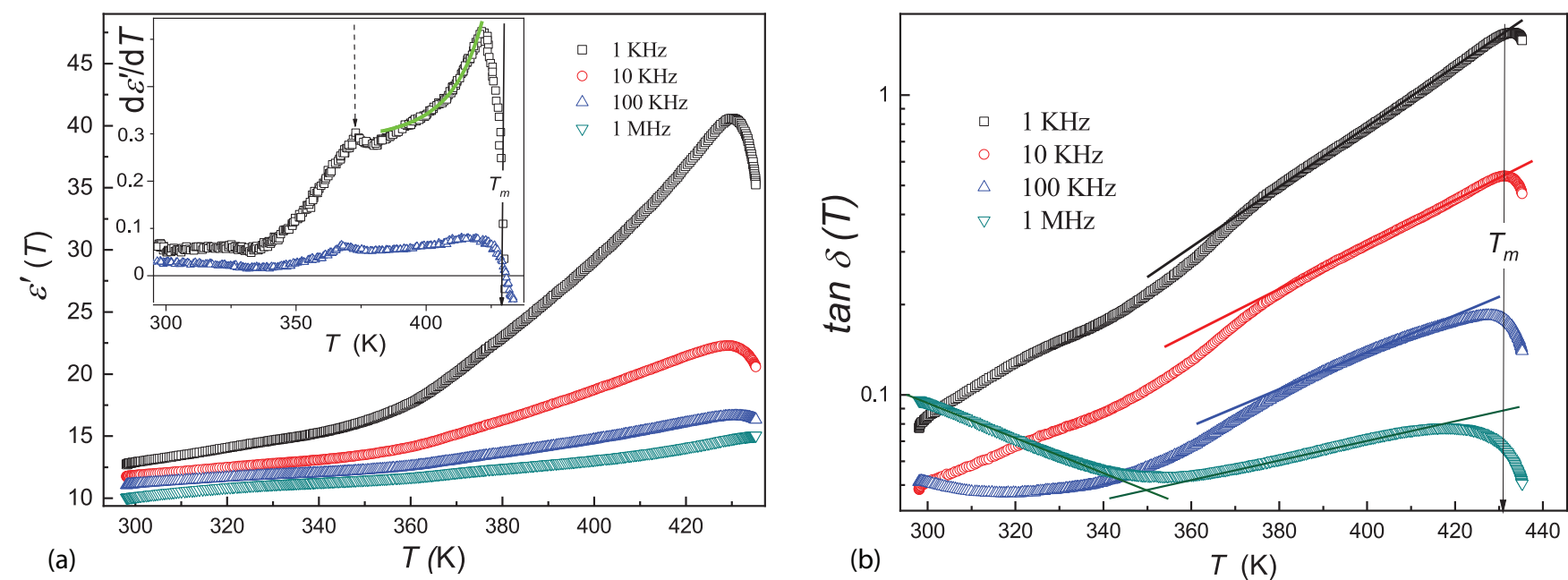

Fig. 2. (a) The temperature behaviour of the real of dielectric permittivity in tested PVDF + BST composites, for selected frequencies. The inset shows derivatives of experimental data from the main part of the plot, revealing hidden phase transitions or crossover temperatures. (b) The temperature behaviour of the dielectric loss in tested PVDF + BST composites, for selected frequencies. Notable is the emergence of domains following the simple linear dependence.


Fig. 3. The temperature evolution of the real and imaginary parts of dielectric permittivity for selected frequencies, showing the impact of BST nanoparticles on dielectric properties.

these composites, the increase of the amount of BST influenced values of the real $\left(\varepsilon^{\prime}\right)$ and imaginary parts $\left(\varepsilon^{\prime \prime}\right)$ of dielectric permittivity but the impact on the primary relaxation time was negligible. Then, its analysis could be focused on the selected, $x=10 \%$ vol. fraction, the concentration of BST in the composite.

One of key features of the glassy dynamics is the non-Debye broadening of the distribution of primary relaxation times $[2,17,18,34]$. As the classical reference serves the Debye type distribution with 'branch' coefficients: $m=n=1$ (Eqs. (7) and (8)) [2,17]. Figures 3 and 4 are focused on the primary relaxation manifesting in temperature $-\varepsilon^{\prime}(T)$ and $\varepsilon^{\prime \prime}(T), f=$ const (Fig. 3) and frequency - related ' $(f)$ and $\varepsilon^{\prime \prime}(f), T$ $=$ const (Fig. 4) scans. The assemble of loss curves $\varepsilon^{\prime \prime}(f, T)$ shows the broadening of the distribution of relaxation times when increasing the concentration of micro-particles (solid lines in Figs. 3b and 4). The broadening of $\varepsilon^{\prime \prime}(f)_{T=\text { const }}$ primary loss curves on cooling $\left(T \rightarrow T_{g}\right)$ is indicated as one of the universal hallmarks of the previtreous behaviour. Notable is also the 'crossover' of the real part of dielectric permittivity $\varepsilon^{\prime}(T)$ on passing $\left(T_{\text {peak }}, f_{\text {peak }}\right): \varepsilon^{\prime}(x=10 \%)>\varepsilon^{\prime}(x=40 \%) \rightarrow$ $T=T_{\text {peak }} \rightarrow \varepsilon^{\prime}(x=10 \%)<\varepsilon^{\prime}(x=40 \%)$, evidenced in Figure 3 (left).

The evolutions of the primary relaxation time for PVDF and BST/PVDF composites are shown in Figure 5. The behaviour of $\tau(T)$ for 'pure' PVDF and BST/PVDF are qualitatively different, regarding the sequence of the 'Arrhenius' and 'Super-Arrhenius' dynamics: SA $\rightarrow T_{g} \rightarrow$ $\mathrm{A}$ for $\mathrm{PVDF}$ and $\mathrm{A} \rightarrow T_{g} \rightarrow \mathrm{SA}$ for BST/PVDF. It is notable that for PVDF and BST/PVDF composite the A/SA and SA/A crossovers are associated with approximately the same value of the glass temperature $T_{g}$. In 


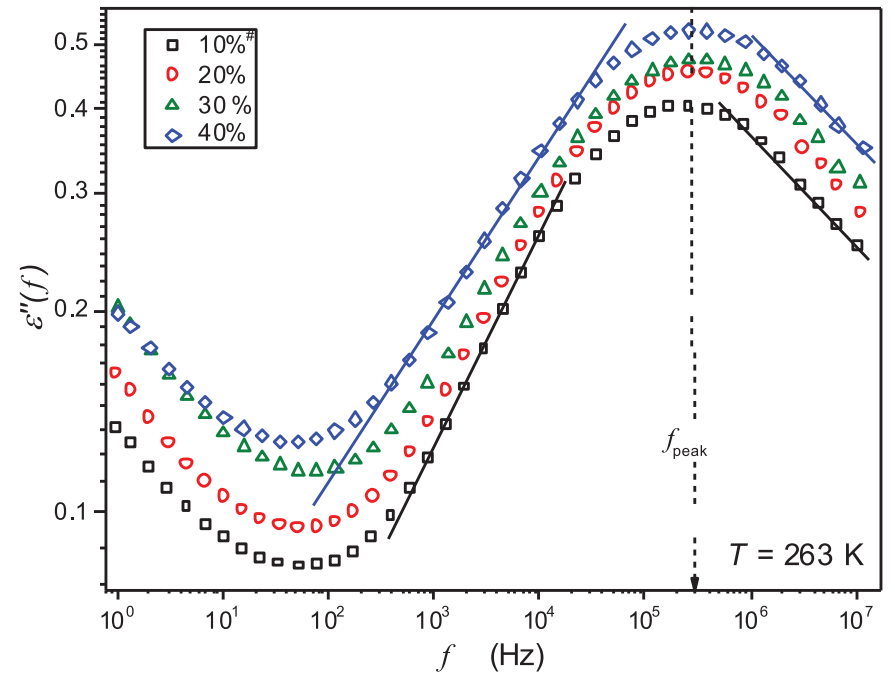

Fig. 4. Dielectric loss curves for the selected temperature, for BST/PVDF composite. Lines show the validity of Jonscher's relations given by equation (15).

the composite, there is also a strong manifestation of the low-frequency Maxwell-Wegner (MW) relaxation process $[2,17,18]$, spanning in the time scale between $\tau_{\text {low }} \sim 10^{-2} \mathrm{~s}$ and $\tau_{\text {low }}\left(T_{g}\right) \sim 5$ s.

So far, for portraying the SA primary relaxation process in PVDF and PVDF based composites the Vogel-FulcherTammann (VFT) equation $[2,17,18,24,25,45]$ was applied $[5-14]$ :

$$
\tau(T)=\tau_{0} \exp \left(\frac{D_{T} T_{0}}{T-T_{0}}\right)
$$

where $T>T_{g}, T_{0}<T_{g}$ is the VFT (extrapolated) singular temperature and $D_{T}$ is the fragility strength being one of metrics of the degree of the SA behaviour (fragility).

It is worth noting that the VFT relation is so common that it is often indicated as the hallmark of the 'universality' of previtreous dynamics in glassy systems $[2,17,18]$. Notwithstanding, in the last decades alternative equations for portraying the previtreous SA behaviour were successfully developed. This is the case of Avramov-Milchev (AM) [26] and Waterton-Mauro (WM, MYEGA) [29,30] equations, without the finite temperature singularity. Another alternative is critical-like dependence, preferable for liquid crystals [31] and plastic crystals [27]. Unfortunately, the residual analysis comparing fitting quality via different relations is weakly conclusive $[2,28,33]$. To overcome this problem, the analysis directly based on the apparent activation energy and its curvature was introduced $[21-23,32,46]: I_{D O}=-d \ln E_{a}(T) / \ln T$ (see Sect. 3). Experimental studies for almost 50 glass formers, ranging from low molecular weight liquids to polymers, resins, plastic crystals, and liquid crystals, showed the 'universal' behaviour of the index [21-23]:

$$
\frac{1}{I_{D O}(T)}=b+a T \text {. }
$$

The condition $I_{D O}^{-1}\left(T=T_{N}\right)=0$ determines the modelfree singular temperature $T_{N}$, which values fairly well correlates with the 'thermodynamic' Kauzmann ideal glass temperature $[22,47]$. Glass formers dominated by different local molecular symmetries are associated with different values of the 'symmetry parameter' $n=-1 / b$, namely: (i) $n \approx 0.2$ is for the 'translational symmetry', (ii) $n \approx 1.5$ is for the 'orientational symmetry' and (iii) $n=1$ is the 'no symmetry' case [21]. The empirical equation (16) enabled the derivation of the new dependence describing the evolution of the structural entropy [21]:

$$
S(T)=S_{0}\left(1-\frac{T_{N}}{T}\right)^{n}, \text { where } n=-1 / b
$$

Its results are shown in Figure 5b, revealing the clear liner behaviour of $1 / I_{D O}(T)$, in the agreement with the 'universal pattern' indicated in references [21-23] and sketched above. However, the obtained values of the coefficient ' $n$ ' are well beyond $n=1$ predicted for the VFT equation (Tab. 1). Consequently, the question arises for the optimal and fundamentally justified portrayal of the $\mathrm{SA}$ dependences presented in Figure 5a. To address this problem, one can recall the recent discussion by DrozdRzoska [48] who proposed to link the extended structural entropy equation (17) and the Adam Gibbs (AG) model, where the previtreous behaviour is the consequence of cooperatively rearranging regions emerging on approach$\operatorname{ing} T_{g}$. The AG model output equation has the following form [49]:

$$
\tau(T)=\tau_{0} \exp \left(\frac{A \triangle \mu}{T S_{C}}\right)
$$

where $\Delta \mu$ defines that the free-energy barrier between cooperatively rearranged regions and $S_{C}$ is the configurational entropy related to the difference between the entropy of the metastable disordered supercooled system and the stable crystal state.

Substituting equation (17) into equation (18) one obtains [48]:

$$
\begin{aligned}
& \tau(T)=\tau_{0} \exp \left(\frac{A \triangle \mu}{T S_{0}\left(1-T_{N} / T\right)^{n}}\right) \\
& =\tau_{0} \exp \left(\frac{A \triangle \mu / S_{0}}{T\left(1-T_{N} / T\right)^{n}}\right)=\tau_{0} \exp \left(\frac{D}{T\left(1-T_{N} / T\right)^{n}}\right) \\
& =\tau_{0} \exp \left(\frac{D T^{n-1}}{\left(T-T_{N}\right)^{n}}\right) .
\end{aligned}
$$

For $n=1$ one obtains the VFT equation (15) with the singular temperature $T_{N}=T_{0}$. Knowing values of $T_{N}$ and $n$ from the analysis presented in Figure $5 \mathrm{~b}$ one can reduce the final fitting of $\tau(T)$ experimental data $\tau_{0}$ prefactor and $D$ coefficient. It is notable that values of $T_{N}$ and $n$ determined without any model-equation assumed in prior, and the exactly known domain of validity (Eq. (16)). Results of such fitting as shown by solid curves in Figure 5a. Assuming that the fragility strength coefficient in equation (19) has the form as analogous to the VFT equation (15), i.e. $D=D_{T} T_{N}$, one obtains: 

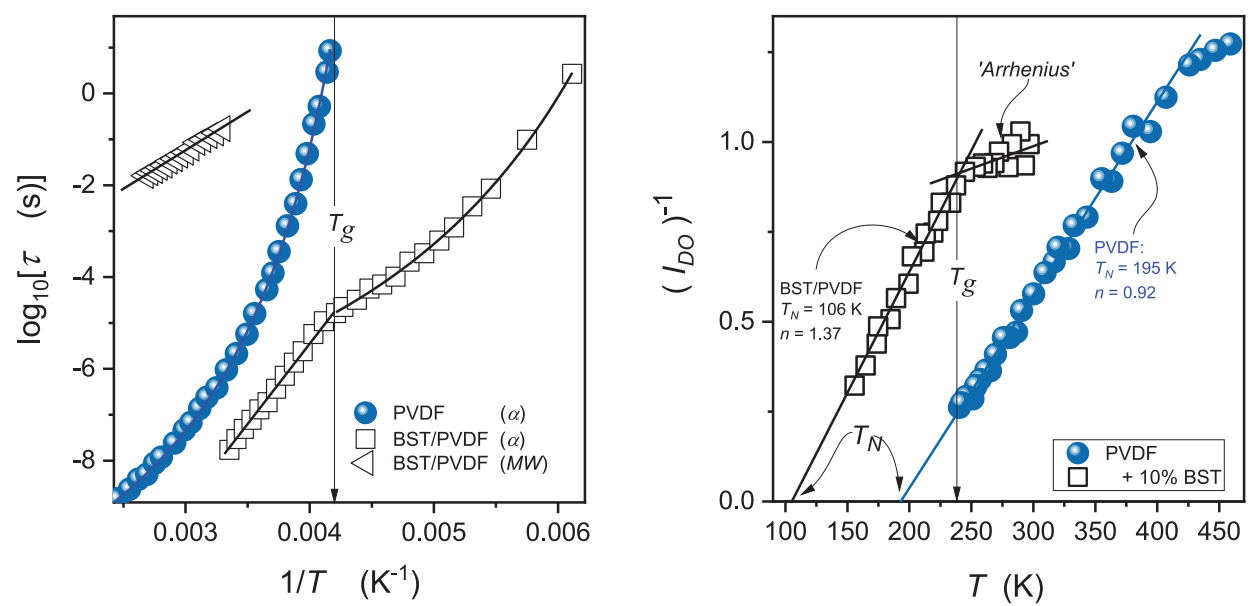

Fig. 5. The behaviour of the primary relaxation time in PVDF and for the 10 vol.\% BST/PVDF composite. The low-frequency process (triangles) is also shown. The evolution of reciprocals of the activation energy temperature index. Values of glass temperature $T_{g}$, the singular temperatures $T_{N}$ and the coefficient $n=-1 / b$ are indicated.


Fig. 6. The temperature evolution of the real and imaginary part of dielectric permittivity, with the focus on the surrounding of the melting temperature $T_{m}$ for BST/PVDF composite. The lower inset in Figure 6a shows the possible description via the Curie-Weiss law. The upper inset in Figure 6a presents the results of the derivative analysis, where the solid (black) curve is for $\varepsilon^{\prime}(T) \sim 1 /\left(T-T^{*}\right)^{4}$. The semi-log scale in the inset of Figure $6 \mathrm{~b}$ validate the portrayal via $\varepsilon^{\prime \prime}(T)=C \exp \left( \pm b_{ \pm} T\right)$ near $T_{m}$.

- PVDF: $\tau_{0}=1.66 \cdot 10^{-12} \mathrm{~s}, D_{T}=6.6$ and $T_{N}=$ $195 \mathrm{~K}, n=0.92$ for $T>T_{g}$

- BST/PVDF: $\tau_{0}=15 \cdot 10^{-9} \mathrm{~s}, D_{T}=7$ and $T_{N}=$ $106 \mathrm{~K}, n=1.32$ for $T<T_{g}$

It is notable that that for the VFT equation the coefficient $n=1$, i.e. relatively close to the value obtained in PVDF for $T>T_{g}$. For the BST/PVDF composite, the SA process developing for $T<T_{g}$ is associated with the value of the coefficient well beyond the VFT pattern and close to the 'orientational' one $(n \approx 1.5)$. This can be correlated with presumably relaxors' nature of the relaxation process in this region, linked to BST ferroparticles.

Figure 6 focuses on the issue of the hypothetical pretransitional effect in the surrounding of the melting temperature $T_{m}$ in BST/PVDF composite, discussed for 'pure' PVDF. Figure 5a shows the behaviour of dielectric constant in BST/PVDF composite from the near room temperature, up to $523 \mathrm{~K}$, i.e., well above melting. The lower inset shows the reciprocal of experimental data from the plot for $T_{m}+10 \mathrm{~K}$, showing the evidence for the Curie-Weiss type behaviour $\varepsilon^{\prime} \propto 1 /\left(T-T^{*}\right)$ for $T<T_{m}$ and $\varepsilon^{\prime} \propto 1 /\left(T^{* *}-T\right)$ for $T>T_{m}$ and $T^{*}=$ $T_{m}+\triangle T^{*}, T^{* *}=T_{m}-\triangle T^{* *}$ stands for hypothetical continuous phase transitions and $\triangle T^{*}, \triangle T^{* *}$ are metrics for the discontinuity of the transition associated with melting in BST/PVDF composite. Strong and long-range is the pretransitional anomaly evidenced in the upper inset of Figure 6a: $\varepsilon(T) / d T \sim 1 /\left(T-T^{*}\right)^{3.8}$ and $\triangle T^{*} \approx 22$ $\mathrm{K}$. It is also notable that PVDF on cooling for $T<T_{m}$ the plot $\varepsilon(T) / d T$ reveals the extra transition/crossover at $T=372.6 \mathrm{~K}$. It is indicated by the dashed arrow in the inset in Figure 2a. This feature is absent for BST/PVDF composite (see the inset in Fig. 6a).

The comparison of Figures 2a and 6 shows that 'pretransitional effects' of $\varepsilon(T)$ and $\varepsilon(T) / d T$ for $T \rightarrow T_{m}$ in 
PVDF and BST/PVDF have the same form, although the range of the 'anomaly' is broader for BST/VDF composite. Figure $6 \mathrm{~b}$ presents the temperature behaviour $\varepsilon^{\prime \prime}(T)$ from the room temperature up $250 \mathrm{~K}$, in the semilogarithmic scale. The inset in Figure $6 \mathrm{~b}$ focuses on the surrounding of the melting temperature, showing the validity of the portrayal via the relation $\varepsilon^{\prime \prime}(T)=C_{ \pm} \exp \left( \pm b_{ \pm} T\right)$. It is worth stressing above such dependence was noted when passing the maximum loss, i.e., it was related to relaxation processes. However, the behaviour presented in Figure 6b it is associated with the vicinity of the phase transition at $T=T_{m}$.

\section{Conclusions}

Results presented above show the unusual impact of the addition of BST micro particles on the PVDF polymer. Particularly worth stressing is the 'reverse' sequence for the Arrhenius/Super-Arrhenius dynamics: $\mathrm{SA} \rightarrow T_{g} \rightarrow \mathrm{A}$ in PVDF and $\mathrm{A} \rightarrow T_{g} \rightarrow \mathrm{SA}$ for BST/PVDF. It is notable that for $T>T_{g}$ the simple Arrhenius evolution of the primary relaxation time is associated with the complex non-Debye distribution of relaxation times. There is a coincidence between the glass temperature $T_{g}$ in PVDF and the crossover temperature $T_{S A}$. All these indicate the 'feedback' interaction between PVDF polymer and BST particles: it very strongly manifests for $\tau(T)$ evolution, but it is also notable for $\varepsilon^{\prime}(T)$ behavior. For $T>T_{g}$ the guest-host interactions in BST/PVDF uniforms the freedom of segmental motions in PVDF, leading to the simple Arrhenius evolution of relaxation times, with the same activation energy in the broad temperature range. It is particularly notable that in the composite the relaxation-type SA behaviour associated with BST particles develops only for $T<T_{g}$. This can mean that above $T_{g}$ the electric field from ferroelectric BST particles 'uniformed' PVDF segmental motions, but the coupled, feedback action also occurred: nanodomains within BST microparticles were blocked by the ferroelectric surrounding of the host (PVDF). When cooling below $T_{g}$ molecular interaction within PVDF is qualitatively strengthened, what causes its finale complete solidification. However, this fact also causes the break in the influence of the host (PVDF) on the guest (BST), and the relaxor-type dynamics can emerge at $T_{g}$ further develop on cooling. We would like to stress the role of the innovative activation energy temperature index, which finally led the test of the new equation for $\tau(T)$ dependence describing the glassy SA behaviour (Eq. (5)). It is generally expected that melting, including polymers, is the first order, discontinuous, transition without pretransitional phenomena characteristic for 'universal' continuous phase transition. Results presented above showed that this is not the case of PVDF and BST/PVDF composites, regarding both $\varepsilon^{\prime}(T)$ and $\varepsilon^{\prime \prime}(T)$. It seems that such behaviour is associated with the para/ferro- electric nature of PVDF and BST what can lead to the appearance of fluctuations heterogeneities, possible to be detected via BDS spectroscopy. None of the facts recalled above was reported for similar systems, so far. This can be associated with the new experimental methodology implemented in the given report. We would also like to stress the significance of results for the physics of phase transitions (melting) and the physics of the glass transition (unusual dynamics). Both findings address grand challenges in recalled areas. Finally, we would like to attract the attention to the finding that in the relaxation and phase transition domains $\varepsilon^{\prime \prime}(T) \propto \exp (b T)$, what can be important for the analysis of experimental data in complex dielectrics.

The authors from IHPP PAS Poland (S.S., S.J.R., E.P.) were supported by the NCN OPUS grant (ref. 2016/21/B/ST3/02203, headed by Aleksandra Drozd-Rzoska). Chinese co-authors were supported by the National Natural Science Foundation of China (No. 51672219), the International Cooperation Foundation of Shanaxi Province, China (No.2017KW-025).

\section{Author contribution statement}

K.Z. and F.G. prepared studied materials, characterized by DSC and XRD methods. S.S., A.D.R., S.J.R. performed dielectric studies, analyzed obtained data and wrote a main part of this paper. E.P. and M.S. discussed analyzed results and the manuscript.

Open Access This is an open access article distributed under the terms of the Creative Commons Attribution License (http://creativecommons.org/licenses/by/4.0), which permits unrestricted use, distribution, and reproduction in any medium, provided the original work is properly cited.

\section{References}

1. D. Kennedy, Science 309, 83 (2005)

2. K.L. Ngai, Relaxation and Diffusion in Complex Systems (Springer, Berlin, 2011)

3. K. Januchta, E. Youngman, A. Goel, M. Bauchy, S.L. Logunov, S.J. Rzoska, M. Bockowski, L.R. Jensen, M.M. Smedskjaer, ACS Chem. Mater. 29, 5865 (2017)

4. A. Ninarello, L. Berthier, D. Coslovich, Phys. Rev. X 7, 021039 (2017)

5. T. Furukawa, Adv. Coll. Int. Sci. 71-72, 183 (1997)

6. R. Gregorio, E.M. Ueno, J. Mat. Sci. 34, 4489 (1999)

7. J. Kaszyn'ska, B. Hilczer, P. Biskupski, Polym. Bull. 68, $1121(2012)$

8. V. Sencadas, S. Lanceros-Mendez, R. Sabater, I. Serra, A.A. Balado Gomez, J.L. Ribelles, Eur. Phys. J. E 35, 41 (2012)

9. E. Ozkazan, H.Y. Guney, T. Oskay, E. Tarcan, Appl. Polym. Sci. 109, 3878 (2008)

10. L. Wang, F. Gao, J. Xu, K. Zhang, M. Wang, M. Quin, High Volt. 1, 158 (2016)

11. J. Martín, A. Iturrospe, A. Cavallaro, A. Arbe, N. Stingelin, T.A. Ezquerra, C. Mijangos, A. Nogales, ACS Chem. Mater. 29, 3515 (2017)

12. C.V. Chanmal, J.P. Jog, eXPRESS Polym. Lett. 2, 294 (2008)

13. H. Rekik, Z. Ghallabi, I. Royaud, M. Arous, G. Seytre, G. Boiteux, A. Kallel, Compos. Part B 45, 1199 (2013) 
14. V.K. Prateek Thakur, R.K. Gupta, Chem. Rev. 116, 4260 (2016)

15. S. Starzonek, S.J. Rzoska, A. Drozd-Rzoska, K. Czupryn'ski, S. Kralj, Phys. Rev. E 96, 022705 (2017)

16. M. Gluszek, A. Antosik, M. Szafran, S.J. Rzoska, M. Zalewski, E. Pawlikowska, S.J. Starzonek, Non-Cryst. Solids 471, 91 (2017)

17. F. Kremer, A. Schoühals, Broad Band Dielectric Spectroscopy (Springer. Berlin, 2003)

18. E. Donth, The Glass Transition: Relaxation Dynamics in Liquids and Disordered Materials (Springer, Berlin, 2001)

19. C.A. Angell, Strong and Fragile Liquids, in Relaxations in Complex Systems, edited by K.L. Ngai, G.B. Wright (Natl. Tech. Inf. Serv., Springfield, 1985)

20. R. Boehmer, K.L. Ngai, C.A. Angell, J.D. Plazek, J. Chem. Phys. 99, 4201 (1993)

21. J.C. Martinez-Garcia, S.J. Rzoska, A. Drozd-Rzoska, J. Martinez-Garcia, Nat. Commun. 4, 1823 (2013)

22. J.C. Martinez-Garcia, S.J. Rzoska, A. Drozd-Rzoska, J. Martinez-Garcia, J.C. Mauro, Sci. Rep. 4, 5160 (2014)

23. J.C. Martinez-Garcia, A. Rzoska, S.J. Drozd-Rzoska, S. Starzonek, J.C. Mauro, Sci. Rep. 5, 8314 (2015)

24. G.S. Fulcher, J. Am. Ceram. Soc. 8, 339 (1925)

25. G. Tammann, J. Soc. Glass Technol. 9, 166 (1925)

26. I. Avramov, J. Non-Cryst. Solids 351, 3163 (2005)

27. A. Drozd-Rzoska, S.J. Rzoska, S. Pawlus, J.L. Tamarit, Phys. Rev. E 73, 224205 (2006)

28. R. Richert, Physica A 287, 26 (2000)

29. J.C. Mauro, Y. Yue, A.J. Ellison, P.K. Gupta, D.C. Allan, Proc. Natl. Acad. Sci. 106, 19780 (2009)

30. S.C. Waterton, J. Soc. Glass Technol. 16, 244 (1932)

31. A. Drozd-Rzoska, J. Chem. Phys. 130, 234910 (2009)
32. T. Hecksher, A.I. Nielsen, N.B. Olsen, J.C. Dyre, Nat. Phys. 4, 737 (2008)

33. A. Drozd-Rzoska, S.J. Rzoska, Phys. Rev. E 73, 041502 (2006)

34. A.K. Jonsher, Dielectric Relaxation in Solids (Chelsea Diel. Press, London, 1983)

35. K.M. Rabe, Ch.H. Ahn, J.-M. Triscone, Physics of Ferroelectrics: A Modern Perspective. Topics in Applied Physics Series (Springer, Berlin, 2007)

36. V.P. Skripov, M.Z. Faizulin, Crystal-Liquid-Gas Phase Transitions and Thermodynamic Stability (Wiley-VCH, Weinheim, 2006)

37. H. Nishimori, G. Ortiz, Elements of Phase Transitions and Critical Phenomena (Oxford Univ. Press., Oxford, 2015)

38. M.A. Anisimov, Critical Phenomena in Liquid and Liquid Crystals (Gordon \& Breach, Reading, 1992)

39. A. Drozd-Rzoska, S.J. Rzoska, J. Ziolo, Phys. Rev. E 54, $6452(1996)$

40. A. Drozd-Rzoska, S.J. Rzoska, J. Ziolo, Phys. Rev. E 61, $5349(2000)$

41. A. Drozd-Rzoska, S.J. Rzoska, K. Czupryn'ski, Phys. Rev. E 61, 5355 (2000)

42. J. Ziolo, B. Fugiel, J. Pawlik, Ferroelectrics 70, 129 (1986)

43. S.J. Rzoska, Phys. Rev. E 48, 113 (1993)

44. D.V. Matyushov, R. Richert, J. Chem. Phys. 144, 041102 (2016)

45. H. Vogel, Phys. Zeit. 22, 645 (1921)

46. J.C. Dyre, N.B. Olsen, Phys. Rev. E 69, 042501 (2004)

47. W. Kauzmann, Chem. Rev. 43, 219 (1948)

48. A. Drozd-Rzoska, Front. Mater. 6, 103 (2019)

49. G. Adam, J.H. Gibbs, J. Chem. Phys. 43, 139 (1965) 